\title{
Real-Time Adaptive Spectrum Sensing for Cyclostationary and Energy Detectors
}

\author{
Antoni Ivanov, Technical University of Sofia, Bulgaria \\ Albena Mihooska, Aarhus University, Herning, Denmark \\ Krasimir Toncheo, Vladimir Poulkov, Technical University of Sofia, Bulgaria
}

\section{INTRODUCTION}

Multiple spectrum measurement campaigns around the world have shown that even though the frequencies below $6 \mathrm{GHz}$ are very crowded, enough portions of the spectrum remain unutilized most of the time. Even the most heavily employed bands, such as the ones used for television broadcasting and cellular communications, have shown an average occupancy between 25 and 50\% [1]. Together with the ever-increasing growth of connected devices in the scope of the Internet of Things (IoT) concept, these have been the main motivational factors for the intensive research in the field of cognitive radio (CR), which empowered by the abilities of the software-defined radio (SDR) devices, can enable the delivery of IoT services, as they operate together with the incumbent users of the existing wireless networks. Consequently, the CR can be utilized for a variety of applications within IoT and the modern systems for delivery of intelligent services, or the traditional standards for wireless data transmission including mobile networks [2]. Therefore, it can be implemented in diverse appliances and professional equipment for integrated navigation, sensing, and communications.

One of the enabling functions of the CR device (also called a secondary user) is the spectrum sensing, which allows for the detection of unused frequency resources. The CR device must free the spectrum once the incumbent or primary user (PU) starts to use it to avoid causing interference; therefore, the time and accuracy for detecting an empty spectrum slot are a very important condition

Authors' current addresses: A. Ivanov is with Technical University of Sofia, Sofia, 1000, Bulgaria, Email: astivanov@ tu-sofia.bg; A. Mihovska is with BTECH, Aarhus BSS, Aarhus University, Herning, 7400, Denmark; K. Tonchev is with Technical University of Sofia, Sofia, 1000, Bulgaria; V. Poulkov is with Technical University of Sofia, Sofia, 1000, Bulgaria, Email: vkp@tu-sofia.bg.

This paper was supported by Contract DN 07/22/2016 of the Bulgarian Research Fund of the Ministry of Education. Research project:"Self-coordinating and Adaptive Wireless Cyber-Physical Systems with Human in the Loop".

Manuscript received May 30, 2017, revised November 1, 2017, and ready for publication December 20, 2017.

Review handled by L. Ligthart.

0885/8985/18/\$26.00 (C) 2018 IEEE of any spectrum sensing algorithm. There are two main parameters, which characterize the spectrum sensing process - the probability of detection $\left(P_{d}\right)$ and probability of false alarm $\left(P_{f a}\right)$. The former defines the probability that the SU will detect the presence of the PU correctly. Alternatively, the probability of miss-detection $\left(P_{m d}\right)$ represents the likelihood that the $\mathrm{SU}$ will not discover the PU. The probability of false alarm describes the possibility that the SU will decide that the PU is present, when in reality the band has not been occupied. An incorrect decision on the spectrum occupancy will result in either interference to the PU (if its presence is not detected) or a loss of opportunity for using unutilized spectrum (in case of a false alarm). That is why the efficiency of the detector is central to most of the research efforts. The traditional spectrum sensing techniques' energy detection, matched filter, cyclostationary detection, and wavelet detection [3] form the basis for most of the known solutions.

In the past decade, the amount of research in relation to spectrum sensing has increased substantially. However, the majority of it is performed theoretically and verified only via computer simulations without testing their performance in practical real-life scenarios. Moreover, no adaptivity or trade-off have been applied in the expressions defining the detection performance in most of the works. There have also been many practical realizations of CR systems ([2], [4]-[17]) with a different range of scientific contributions but they are much fewer in comparison to the works which present solutions verified only via simulations. This highlights the need for more practical implementations. Recently proposed CR applications ([18]-[21]) consider only simple energy detection as the local spectrum sensing method which is the basis for their overall architectures, and in some cases, the role of spectrum sensing has not been considered at all. In addition, there is a substantial evidence in the literature ([22]-[24]) that suggests that the time, which is used for the spectrum sensing process should be adapted to the patterns of the PU transmission, for achieving an optimal trade-off between the sensing accuracy (proportional to the sensing period) and the loss of opportunity for the utilization of the unused spectrum. These facts indicate the need for examinations of robust detectors in real-life environments.

In this article, we explore the details of some practical implementations of energy and cyclostationary detectors, which take into account the specific radio channel impairments (like noise uncertainty and fading), using the Universal Radio Serial Peripheral 
(USRP) hardware platform and the GNU Radio software. Both of these methods have straightforward analytical definitions and do not differ much in terms of implementational complexity. The energy detection method is known to be computationally light but lacking efficiency in very low SNRs [25], [26]. The cyclostationary detector has, in general, the opposite characteristics. The two detectors are included into a framework, which adapts the sensing period by using a precalculated efficiency-accuracy trade-off and a method for estimating the pattern of the PUs operation.

Our novel contributions presented in this article are the following:

1. A performance analysis of real-time practical implementations of known cyclostationary [27] and energy [28] detectors, including Rayleigh fading channel.

2. Method for the estimation of the channel parameters and analysis of the detection performance integrated with the spectrum sensing procedure for an obstructed non-line-of-sight (NLOS) scenario where the receiver has a limited mobility.

3. Functionality for adaptive change of the sensing time through the addition of a simplified method for the estimation of the PUs states and analysis of the efficiency-accuracy trade-off for three different PU configurations implemented in the transmitter.

4. A detailed outline of the characteristics of implementing an adaptive spectrum sensing algorithm using USRP and GNU Radio.

\section{BACKEROUND AND RELATED WORK}

Spectrum sensing algorithms based on energy and cyclostationary detectors and their respective advantages and weaknesses, have been widely researched. In this section, a summary of existing advances in these topics including practical implementations, is presented.

\section{ENERGY DETECTION-BASED SPECTRUM SENSING}

The energy detector proposed in [29] obtains the decision threshold by setting the probability of false alarm as constant and using the standard expression for the constant false-alarm method ((13) in [23]).
$P_{f a}=Q\left(\sqrt{2 \gamma+1} Q^{-1}\left(\bar{P}_{d}\right)+\sqrt{M} \gamma\right)$,

where $\bar{P}_{d}$ is the target probability of detection, $\gamma$ is the SNR, M is the number of observed samples and $Q($.$) is the complementary$ distribution function of the Gaussian variable, i.e.

$Q(x)=\frac{1}{2 \pi} \int_{x}^{\infty} \exp \left(-\frac{t^{2}}{2}\right)$

It also implements a method for noise variance estimation. Further improvement is achieved by deriving a new threshold, which is built upon the traditional one but depends on the signal-to-noise ratio (SNR) as well [30]. Another approach is proposed in [31] which introduces upper and lower thresholds, which define a "confused region", in which the algorithm attempts to identify whether the signal power is closer to the noise-only region or the PU signal region. In [32], the threshold is bound and the bounds are dynamically defined on the basis of the noise uncertainty in order to increase the efficiency, both, in terms of $P_{d}$ as well as $P_{f a}$. This proposal for an energy detector (ED) outperforms the traditional forms of two other more complex spectrum sensing techniques. The algorithm in [33] combines an energy and a cyclostationary detectors to improve the overall performance by using the former in high SNR and the latter in low SNR. The above-mentioned solutions all follow the traditional derivation of the performance of the ED, which assumes a Gaussian distribution of the received signals in additive white Gaussian noise (AWGN) channel and very low SNR (between -20 and $0 \mathrm{~dB}$ ). They do not implement adaptivity in terms of the sensing time and only study the detection accuracy. A generalized ED with adaptations for several fading channels is derived in [34]. The analysis in this article focuses on assessing the performance of the detector in the case of diversity reception and cooperation between multiple SUs. Alternatively, accurate spectrum occupancy evaluation can be achieved through cooperative spectrum sensing and a decision fusion center with multiple antennas, which processes the observations of the SUs [35]. The authors in [35] devise multiple access channel protocols which take advantage of the multiple-input multiple-output concept to obtain more reliable spectrum sensing decision.

In this article, we assume the ED proposed in [28]. It is suitable for practical implementations, like ours, because it presents deri- 
vations of the closed form expressions for the probability of missdetection for different fading channels. In comparison to [34], the detector is made more resilient by the introduction of a bounded decision threshold. The bounds are chosen to optimize the ratio of $P_{m d}$ and $P_{f a}$. The noise uncertainty is also accounted for by integrating the $P_{m d}$ and $P_{f a}$ over its interval.

\section{CYCLOSTATIONARY DETECTION-BASED SPECTRUM SENSING}

Cyclostationary detectors have received much attention in the scientific community. Most of them ([36], [37], [38]) define the test statistics as the sum of the Spectral Correlation Functions (also called Cyclic Spectral Densities) for one (single-cycle detector) or more (multicycle detector) cyclic frequencies (CFs) of the PU signal. It is also often the case that the derivation for $P_{f a}$ and $P_{d}$ for AWGN channel is used. The solution in [36] proposes a cooperative spectrum sensing scenario, in which each SU estimates the Spectral Correlation Function (SCF) of one of the cyclic frequencies and the central SU combines the results to decide whether the band was occupied or not. Such an approach decreases the computational complexity of the operation. The authors in [37] compared the same kind of cyclostationary detector against an ED and a matched filter, showing that the cyclostationary detector outperforms the other two. A hybrid sensing method is introduced in [38]. The ED is applied initially, and then, if the decision is not clear, the cyclostationary detection will be employed. In this hybrid method, the noise is differentiated from the signal, by checking whether the SCF is 0 for a specific CF.

All the papers mentioned above only consider the detection performance in an AWGN channel and do not include the adaptable sensing time.

A recent study in [27] shows that the test statistics could be obtained using the Cyclic Autocorrelation Function (CAF), which is directly derived from the autocorrelation function. This makes the detection algorithm more computationally efficient than such that utilize the SCF because it is the Fourier transform of the CAF. The effectiveness of the method could be further increased by implementing the process as a moving average (sliding correlation) rather than the classic one. For that reason, the authors termed the method "single-cycle detector with sliding correlation" (SCD-SC). The closed-form expressions for the $P_{f a}$ and $P_{d}$ are derived. By integration over the distribution of the channel coefficient, the expression for the $P_{d}$ is applied for the flat-fading case. Due to these advantages in comparison to the other alternatives ([36], [37], [38]), we have chosen the same algorithm for our implementation of the cyclostationary detection scheme.

\section{TRADE-DFF BETWEEN THE SPECTRUM SENSING EFFICIENCY AND ITS ACEURACY}

There are some works ([22], [23], [24]) which examine the potential problems related to how long the sensing period should last so that an opportunity to utilize an unused chunk of the spectrum may not be lost, and also what the length of the transmission period of the SU should be, so that the chance that it will create interference to the returning PU, is minimal. All of these works consider computer simulations of a traditional ED, which operates within a scheme which incorporates the sensing and the transmission periods of the SU. An expression, which takes into consideration the $P_{d}$ and $P_{f a}$, the probability that the PU is active/absent from the band, and the distribution of the states of the PU (present/absent in the band) has been developed. In [22], the proposed framework uses the result given by the detector and combines it with the distribution of the states of the PU, as a renewal process function model. The optimal value of this function was found for a defined length of the frame of the SU. Another alternative is presented in [23]. The distribution of the PU states is not included into the efficiency-accuracy trade-off expression, only the assumed a priori probabilities that the PU does or does not occupy the band. The conclusion is similar to the one made in [22] - the results yield one optimal value for the sensing time for the specific parameters (e.g., the number of slots, into which the frame is divided and the duration of the frame). In [24], the efficiency-accuracy trade-off is obtained by introducing a bound, taking into account the expected SU interference and whether it would be tolerable or not, as well as the expected lost opportunity for the SU to utilize the unused spectrum due to false alarms. A spectrum sensing framework is built around these parameters but the essence here is that they are mostly dependent on the characteristics of the PU states. Therefore, as evident from the results, there would exist only one optimal sensing time for each set of PU parameters and the performance of the detector does not affect the result. This outlines the importance of the definition of the PU states and their estimation at the SU. It also suggests that there may not be a need to solve the trade-off expression during the spectrum sensing process but that can be done a priori. Thus, the already known optimal sensing time can be defined as a constant if the parameters of the PU do not change, or it can be chosen from a set of values, in the case when these vary (which is likely in a realworld scenario). The adaptive approach proposed in this article is based on [22] and it appoints the optimal sensing time depending on the estimated probability of the PU being present in the spectrum band as described in detail in later sections of this article.

\section{PRACTICAL IMPLEMENTATIONS OF ENERGY AND CYCLISTATIONARY DETECTORS}

A large number of the existing reports on practical realizations of spectrum sensing algorithms have only emerged in the last few years. In general, the SDR platform used for such experiments is the USRP one because of its flexibility in producing signals with various modulations and bandwidths. Similar flexibility coupled with processing speed can be achieved using field-programmable gate array (FPGA) platforms, which is why they have also been used for spectrum sensing applications in the recent years [12][16]. Several USRP-based implementations of energy and cyclostationary detectors as reported in [4]-[9] examine the scenario where one USRP is the PU transmitter, while the other is the SU receiver, which implements the spectrum sensing function.

The solution in [4] specifies the desired decision threshold and the number of samples for the test statistic manually from the ob- 
tained graphical results for the desired values of $P_{f a}$ and $P_{d}$. With the use of an analytic expression, the sensing time is precalculated on the basis of the known PU parameters. In a similar manner, in [5], the bounds of the threshold are chosen empirically and the probabilities of miss-detection and of false alarm are compared for different thresholds within the range until an optimal value was found. This study examined signals with various frequency ranges, which was enabled by the use of the more advanced USRP B200 model. A combination of the two types of energy detectors was presented in [6]. The edge ED integrated into the traditional ED was used to locate the edges of the active subcarriers in the Power Spectrum Density in order to discriminate the occupied from the available portions of the spectrum. This method is less dependent on whether the decision threshold of the standard detector is chosen accurately or not. By using a signal generator as a PU, the received power and SNR can be estimated precisely because the generator defines clearly the transmitted power [10], [11], while this may not be so simple when the PU is implemented via a USRP. In [10], the received signal is obtained by calibration with the generator. The noise uncertainty, though studied extensively in the works involving solely computer simulations, has not been considered in the majority of the practical realizations. The authors in [11] included the noise uncertainty into the standard expressions for $P_{d}$ and $P_{f a}$ and added bounds to the threshold, which were dependent on the desired $P_{f a}$. This study further uses a signal generator and examines the cooperative spectrum sensing case with multiple USRP SUs to increase the efficiency of the detector.

The detector implemented in [7] compares three methods, which calculate the test statistics of the received signal using only the autocorrelation function. Such an approach makes the process less computationally intensive than a cyclostationary detector and requires only the cyclic prefix length and the Fast Fourier Transform (FFT) size of the PUs orthogonal-frequency division multiplex (OFDM) signals to be known. In this form, however, the detector is not applicable to other types of signals and the threshold can be defined only empirically and for the specific case.

An example of the implementation of a cyclostationary spectrum sensing is provided in [8]. The method uses the symmetry property of the CAF to obtain a decision. A scheme for compressed sensing is also applied to increase the computational efficiency of the calculations. The proposed detector is blind, in the sense, that it does not require any prior information about the signal, neither were the $P_{d}$ and $P_{f a}$ determined analytically, which makes the detector less flexible for introducing adaptability into it. In [9], the same cyclostationarity method was proposed and compared to a modified ED method, which compared the energy of sequential chunks of the gathered samples until above the upper boundary of a threshold, or below the lower boundary. This approach can improve the speed of the ED, however the method did not consider a fading scenario.

A comprehensive survey on implementation of cyclostationary detectors using the FPGA platform is reported in [12]. The examined detectors show robustness in low SNR under a substantial number of samples (over half a million). In spite of this the detection time is small because of the processing speed of the FPGA. A more recent study [13] using the same type of platform shows that cyclostationary detectors can be used for reliable signal recog- nition under specific radio impairments such as carrier frequency offset, phase noise and others even though some of them lead to a noticeable decline in the detection performance. Similar results are achieved in [14] but for a signal with much higher bandwidth. A novel algorithm for cyclostationary detection was introduced in [15]. It employs the concept of tunneling which provides the possibility to detect and classify different types of signals even though they are under-sampled. That is why it can achieve faster and more accurate processing than the typical cyclostationary method if modulation classification is necessary. The authors in [16] proposed a cognitive radio OFDM system implemented on a FPGAbased SDR card equipped directly to a computer. This device supports simultaneous transmission and reception which allows the SU to utilize the portions of the spectrum which are available.

\section{COGNITIVE RADIO ARCHITECTURES}

Other works, such as [18]-[21] have proposed complete architectures for cognitive communications and spectrum sharing where the spectrum sensing is an essential functionality.

The study in [18] examined a framework for spectrum sharing which enables a base station with both licensed networks and cognitive radio capabilities, to allocate resources to the two types of users. A simulation of video transmission within a single cell with primary and secondary users served by the same base station is investigated. The emphasis is on developing a system for optimal resource allocation between the users of the two radio access technologies, which minimizes the cost (in energy consumption) for the CR terminals. A simple ED model is assumed for the spectrum sensing function, but it is adaptive in the sense that the sensing duration is determined according to the priority of the video packets. Therefore, this adaptation is not a part of the sensing function itself but is performed together with the optimization of the rest of the parameters of the framework.

Another application of spectrum sensing for dynamic spectrum access was presented in [19]. The authors studied the opportunity for a Wi-Fi network to utilize the spectrum used in general for radar transmission. A framework was proposed that employs the random idle periods, which generally would occur in a Wi-Fi system. The study is concentrated on finding the balance between the amount of transmitted packets and the delay in detecting a radar pulse (which can be detected only during the idle periods of the Wi-Fi). It is assumed that detection will always be correct and so the spectrum sensing function is not examined at all.

Cooperative spectrum sensing with emphasis on security was studied in [20]. Threats to the accurate spectrum assessment, of different kinds, are considered and the performance of the proposed solution for distributed cooperative decision-making is verified via simulations. The shadowing effect is considered for determining the efficiency of the overall cooperative detection scheme. The SU nodes perform local spectrum sensing using the traditional energy detector.

Lagunas et al. [21] proposed a resource allocation framework, which achieves spectrum sharing between Fixed Satellite Service terminals (secondary users) and terrestrial microwave links (primary users). The study centered on carrier, power and bandwidth allocation under interference constraints for the transmissions of 
the PU/SUs (for the downlink case, the interference created by the PUs to the SUs is regarded while for the uplink case, it is the opposite). Spectrum sensing is not considered as a separate function inside the framework, but a type of energy detection is implemented as a part of its main decision-making algorithm.

These publications demonstrate relevant application scenarios, but they do not consider the actual detection model in realistic environments. Detectors of weak PU signals, which have been validated via practical implementations, are necessary.

A spectrum sharing architecture implemented using a Wi-Fi/ Long-Term Evolution testbed is examined in [17]. It includes software which controls the operation of the system and similarly to the previously mentioned solutions, it does not focus on the process of spectrum sensing but utilizes a simple ED.

So far there has only been one experimental complete CR implementation [2] which has been tested in the premise of a realworld primary network. The CR network operates under specific power constraints to ensure that the incumbent users will not experience quality of service degradation. A mechanism is implemented which allows the CR base stations to be notified when a PU needs to return to a portion of the spectrum so that the CR network can be reconfigured to vacate it. Thus, the spectrum sensing functionality is simplified.

The fact that these architectures and implementations consider primarily, simple spectrum sensing techniques further highlights the necessity of evaluation of more complex and accurate detectors implemented in realistic scenarios.

\section{SYSTEM MODEL}

\section{CYCLISTATIONARY DETECTION-BASED SPECTRUM SENSING}

Although the cyclostationary detector requires much greater computational complexity than the ED, its advantage is that as the noise does not exhibit cyclostationary properties, the signal could be detected even if it is very weak. Results from various research studies ([7], [8], [9] [36], [37], [38]) have shown that the method has very good detection accuracy in low SNR (the range -20 to 0 $\mathrm{dB}$ is of particular interest). In many of them, the SCF is compared against the decision threshold $\lambda$, however, in our implementation we use the CAF. The estimated CAF for a specific cyclic frequency $\alpha$ and a time lag $\tau$ is defined as in [27]:

$\hat{R}_{x}^{\alpha}(\tau)=\frac{1}{M} \sum_{n=0}^{M-1} R_{x}(n, \tau) e^{-j 2 \pi \alpha n}$,

where $M$ is the observed number of samples and $R_{x}(n, \tau)$ is the autocorrelation function of the signal $x(n)$ with period $n$ equal to $T_{s}$ (the signal period).

In order to reduce the computational complexity of the operation, the authors in [27] proposed a small modification, which establishes the overall solution as a single-cycle detector with sliding correlation (SCD-SC). The sliding window has a length of $N$ samples.

$\hat{R}_{x}^{\alpha}(\tau)=\frac{1}{M} \frac{1}{N} \sum_{n=0}^{M-1} \sum_{i=n}^{n+N-1} x(i) x^{*}(i+\tau) e^{-j 2 \pi \alpha n}$.
Thus, the test statistic is given by (3):

$\Lambda(\alpha)=\left|\hat{R}_{x}^{\alpha}\right|^{2} \gtreqless \lambda$.

The detector decides that the spectrum is occupied by the PU $\left(H_{1}\right)$ if the statistic is larger than the threshold $\lambda$, otherwise it is available for utilization by the SU $\left(H_{0}\right)$. The derivations of the probabilities of detection and of false-alarm are based on the assumptions that the noise is AWGN with zero mean and variance while the PU signal is a random variable with zero mean and variance $\sigma_{w}^{2}$ while the PU signal is a random variable with zero mean and variance $\sigma_{s}^{2}$.

The probability of false alarm is defined in the following way:

$P_{f a}=e^{-\lambda \frac{M N}{2 \sigma_{w}^{4}}}$

Therefore, we can set the $P_{f a}$ as a constant and calculate the decision threshold as in (5):

$\lambda=-\frac{2 \sigma_{w}^{4} \ln \left(P_{f a}\right)}{M N}$.

We should note, here, that because of our implementation, the variance of the noise is constant, and therefore, the threshold value does not vary dramatically. Thus, utilizing a constant false-alarmrate method is equivalent to taking the threshold as a parameter, on the basis of which the $P_{f a}$ can be calculated. In comparison, the dependency between the threshold and $P_{f a}$ in the case of the traditional energy detector [23] is similar but there, the number of the observed samples is directly related to the received SNR, and therefore the threshold is changing (it is inversely proportional to the SNR).

Finally, we use the derived expression for the probability of detection in a Rayleigh flat-fading channel. Here, it should be considered on whether the common assumption about the distribution of the channel attenuation coefficient, is to be employed or not. Preliminary measurements allowed us to estimate the distribution of $h$ (and consequently, for $h^{2}$ ) for our setting. It was verified that the Rayleigh distribution is suitable for the gathered data by examining the received samples using the Bayesian Information Criterion to find the best fit for them. Then we find the mean of the distribution $\mu$. With it added, the expression for $P_{d}$ from [27] is written as

$$
\begin{aligned}
& P_{d}=\int_{\min (h)}^{\max (h)} Q_{1}\left(\frac{\gamma_{f}}{v_{f}}, \frac{\sqrt{\lambda}}{v_{f}}\right) \frac{e^{\frac{-h^{2}}{\mu}}}{\mu} d h^{2}, \\
& v_{f}=\frac{\sigma_{w}^{2}+\sigma_{s}^{2} h^{2}}{\sqrt{M N}} ; \quad \gamma_{f}=\left|\hat{R}_{x}^{\alpha}(\tau)\right| h^{2} .
\end{aligned}
$$

\section{CHANNEL ESTIMATION TECHNIQUE}

For calculating the integral (6) over the distribution of $h^{2}$ we need to estimate the channel attenuation at each detection instance. In order to estimate $h$, the following procedure is implemented. We obtain the initial value of $h$ by using (5) from [39]: 
$\bar{h}=\frac{p-P_{n}}{\sqrt{2 E_{p}}}$

where $E_{p}$ is the energy of the transmitted signal, $p$ is the received signal sample when the transmitter is close to the receiver and $P_{n}$ is the noise (power measured when there is no signal in the band).

Since we concentrate on the spectrum sensing method itself and its ability to discover weak signals, we generalize the transmitted signal and do not transmit any specific information by it. Therefore, there is no need to make any discrimination between the information and pilot signals. The transmitter uses the same signals with the same cyclic characteristics, modulation, and output power. Thus, it is not needed to create a transmission cycle of pilot and information signals, we simply aim to recognize the presence of a particular type of signal. Based on this, we can build an estimator very similar to the one proposed in [39]. A part of the samples $k$ gathered during the sensing period is divided into ten chunks (which contain $M_{k}$ elements) and thus, by performing the estimation 10 times, the vector for $h$ is formed. By generalizing (6) from [39], as suggested in [40], we get

$\tilde{h}_{k}=\tilde{h}_{k-1}+\frac{\sum_{k=0}^{M_{k}-1} p_{k}}{2 M_{k}}$.

The minimum and maximum values of $\tilde{h}$ in the vector are taken as boundaries of the integral. All of the gathered samples (including those used for estimation) are used in the calculation of the CAF, so they are fully utilized.

\section{ENERGY DETECTION-BASED SPECTRUM SENSING}

The energy detector is a simple and often applied detection technique for spectrum sensing. It is based on comparing the average of the squared of the received energy samples $y(k)$ during the sensing period $t_{s}$, to a decision threshold $\lambda$ [41]. The test statistic $\Lambda$ is defined as:

$\Lambda=\frac{1}{M} \sum_{n=1}^{M}|y(n)|^{2}$

where $M=f_{s} t_{s}$ is the number of the observed samples and $f_{s}$ is the sampling rate.

If the received energy is greater than the threshold, the detector decides in favor of $H_{1}$, otherwise, $H_{0}$ will be chosen. The hypothesis $H_{0}$ is true when the received sample $y(n)$ is comprised of only noise $w(n)$, while the alternative will be chosen if both the PU signal $s(n)$ (multiplied by the channel coefficient $h(n)$ ) and noise are detected.

$y(n)= \begin{cases}w(n), & \text { then } H_{0} \\ h(n) s(n)+w(n), & \text { then } H_{1} .\end{cases}$

The noise is AWGN with a zero mean and a variance of $\sigma_{w}^{2}$ while the signal's distribution is generalized with zero mean and variance $\sigma_{s}^{2}$. Because we use a large number of samples, we can define the variance in the same way as it is done in [28]. Following these definitions, the authors in [28] have derived the expressions for the probability of miss-detection in various fading channels.
Because of our conclusion that the channel has Rayleigh distribution, we choose the respective expression

$$
\begin{aligned}
P_{m d}^{\prime} & =\frac{1}{2}\left[\operatorname{erfc}(\alpha)-e^{a^{2}} e^{2 a \alpha} \operatorname{erfc}(a+\alpha)\right], \\
a & =\frac{1}{\sqrt{2 M} \gamma} ; \quad \alpha=\frac{M \sigma_{w}^{2}-\lambda}{\sqrt{2 M} \sigma_{w}^{2}},
\end{aligned}
$$

where the SNR is $\gamma=h^{2} \sigma_{s}^{2} / \sigma_{w}^{2}$.

The probability of false alarm is

$P_{f a}^{\prime}=\frac{1}{2} \operatorname{erfc}\left(\frac{\lambda-M \sigma_{w}^{2}}{\sqrt{2 M} \sigma_{w}^{2}}\right)$.

In [28] the noise uncertainty $\rho$ is represented by a uniform distribution in the limits of $\left[\frac{P_{n}}{\rho}, \rho P_{n}\right]$, where $P_{n}$ is the nominal noise power (in our case, the average power when no signal is present in the band). The uncertainty (in the premise where the experiment is performed) is estimated to be $1.6(2 \mathrm{~dB})$ by empirically defining the SNR wall as is described in [25]. The probability density function (PDF) of $\rho$ is

$g_{\sigma_{w}^{2}}(x)= \begin{cases}\frac{1}{\rho P_{n}-\left(P_{n} / \rho\right)}, & x \in\left[\frac{P_{n}}{\rho}, \rho P_{n}\right] \\ 0, & \text { otherwise. }\end{cases}$

By integrating the $P_{m d}^{\prime}$ and $P_{f a}^{\prime}$ over the limits of the function, as is done in the article [28], the effect of the noise uncertainty is accounted for.

$\begin{aligned} P_{m d} & =\int_{\frac{P_{n}}{\rho}}^{\rho P_{n}} P_{m d}^{\prime} g_{\sigma_{w}^{2}}(x) d x \\ P_{f a} & =\int_{\frac{P_{n}}{\rho}}^{\rho P_{n}} P_{f a}^{\prime} g_{\sigma_{w}^{2}}(x) d x .\end{aligned}$

As for the decision threshold $\lambda$, the authors in [28] propose a double-bounded threshold in order for a specific target probability to be achieved for both the probability of miss-detection and the probability of false alarm, at the same time. The lower bound is defined by the threshold $\lambda_{f}^{*}$ which guarantees that the false alarm probability will be below the required value (denoted by $\bar{P}_{f a}$ ).

$\lambda_{f}^{*}=\left(\sqrt{\frac{2}{N}} \operatorname{erfc}^{-1}\left(2 \bar{P}_{f a}\right)+1\right) M \sigma_{w}^{2}$.

The upper bound is defined by the threshold $\lambda_{m d}^{*}$, which guarantees that the miss-detection probability will be below the requirement (the required value is denoted by $\bar{P}_{m d}$ ):

$$
\begin{gathered}
\lambda_{m d}^{*}=\left[1-\sqrt{\frac{2}{M}\left(\frac{\sqrt{\pi} u-\pi^{\frac{1}{4}} \sqrt{b}}{2 a(1-\sqrt{\pi} u)}\right)}\right] M \sigma_{w}^{2} \\
b=4 \bar{P}_{m d} a-2 a-u\left(2 a\left(2 \bar{P}_{m d}-1\right) \sqrt{\pi}+\sqrt{\pi} u-2\right) u=a e^{a^{2}} \operatorname{erfc}(a) .
\end{gathered}
$$


Then, the optimal solution for the threshold $\lambda_{e}^{*}$ is defined as such, for which both the $P_{m d}$ and $P_{f a}$ are within their predefined limits.

$\lambda_{e}^{*}=\left(1+\frac{1}{M \gamma}-\sqrt{\frac{2}{M \pi}}+\sqrt{\frac{2}{M}\left(\frac{1}{\pi}+\sqrt{\frac{2 M}{\pi}} \gamma-1\right)}\right) \sigma_{w}^{2}$.

Finally, the threshold $\lambda$ is determined as below:

$\lambda= \begin{cases}\lambda_{e}^{*}, & \text { if } \lambda_{f}^{*} \leq \lambda_{m d}^{*} \text { and } \lambda_{e}^{*} \in\left[\lambda_{f}^{*}, \lambda_{m d}^{*}\right] \\ \lambda_{f}^{*} \text { or } \lambda_{m d}^{*}, \text { whichever is closer to } \lambda_{e}^{*}, & \text { if } \lambda_{f}^{*} \leq \lambda_{m d}^{*} \text { and } \lambda_{e}^{*} \notin\left[\lambda_{f}^{*}, \lambda_{m d}^{*}\right] \\ \text { no solution, } & \text { if } \lambda_{f}^{*}>\lambda_{m d}^{*}\end{cases}$

\section{EFFICIENCY-ACCURACY TRADE-DFF}

The purpose of this analysis is to find the optimal balance between the accuracy of the sensing process (associated with the sensing time $t_{s}$ ) and the efficiency (time) of the transmission period of the SU so that a better opportunity to utilize the spectrum is achieved. In order to do this, based on [22], [23], [24] we can form a closedform expression for the spectrum opportunity which includes the sensing efficiency $\xi(18)$, the likelihood $\phi$ that the channel occupancy is determined correctly, and the probability mass function $\psi$ of the discrete states (idle/active or ON/OFF) of the PU. The sensing efficiency is defined as the fraction of the frame which is used for the transmission time of the SU

$\xi\left(t_{s}\right)=\frac{T-t_{s}}{T}$

Thus, the trade-off expression for the spectrum opportunity $\eta$ is formulated in [25] as

$$
\begin{aligned}
\eta\left(t_{s}\right) & =\xi\left(t_{s}\right) \phi\left(P_{d}, P_{f a}\right) \psi \\
& =\frac{T-t_{s}}{T}\left(\operatorname{Pr}(O F F)\left(1-P_{f a}\right)+\operatorname{Pr}(O N)\left(1-P_{d}\right)\right) \psi .
\end{aligned}
$$

In order to find the PDF of the alternating states of the PU, we consider that they form a renewal process [22], [25], [42]. The random variables $T_{O N}, T_{O F F}$, and $T_{R P}$ describe the variations of the "ON" state, the "OFF" state, and the renewal process the elements of which are the sums of the respectable states, $T_{i}^{R P}=T_{i}^{O N}+T_{i}^{O F F}$, $i=1,2,3, \ldots, n$. The probabilities that the PU is active/idle are $\operatorname{Pr}(O N)$ and $\operatorname{Pr}(O F F)$, correspondingly.

For the sake of space, the detailed definitions of the renewal process and the specific derivation of the trade-off expressions are not presented here but they can be found in [25]. The efficiencyaccuracy expression is thus formulated

$$
\begin{aligned}
\eta\left(t_{s}\right) & =\frac{T-t_{s}}{T} c\left[\exp \left(-\frac{T-t_{s}}{\mu_{O N}}\right)-\exp \left(-\frac{T-t_{s}}{\mu_{O F F}}\right)\right] \\
& \left(\operatorname{Pr}(O F F)\left(1-P_{f a}\right)+\operatorname{Pr}(O N)\left(1-P_{d}\right)\right), \\
c & =\frac{\left(\mu_{O F F} \mu_{O N}\right)^{3}}{\mu_{O F F}^{2}-\mu_{O N}^{2}} .
\end{aligned}
$$

where $\mu_{O N}^{-1}$ and $\mu_{O F F}^{-1}$ are the means of $T_{O N}$ and $T_{O F F}$, respectively.

We find the optimum value of $t$ by maximizing the function within a specific interval. This interval is defined by the shortest and longest periods, which $t_{s}$ can last within one frame.

\section{ESTIMATION DF THE STATES OF THE PRIMARY USER}

As discussed earlier, for each configuration of PU parameters $\left(\operatorname{Pr}(O N)\right.$ and $\operatorname{Pr}(O F F), \mu_{O N}^{-1}$ and $\left.\mu_{O F F}^{-1}\right)$, there is only one optimal value of the sensing time. Therefore, there is no need for the computationally intensive process of finding it, to be performed at each spectrum sensing iteration. That is why we first estimate the parameters of the PU and then find the trade-off. This way we can set it as a constant for a specific PU configuration. If we define three types of $\mathrm{PU}$ transmission patterns-where $\operatorname{Pr}(O N)>\operatorname{Pr}(O F F)$, $\operatorname{Pr}(O N) \approx \operatorname{Pr}(O F F), \operatorname{Pr}(O N)<<\operatorname{Pr}(O F F)$, we can obtain one optimal $t_{s}$ for each of them. The SU can choose one of the three options based on a simplistic estimation method. In this implementation, we record every decision of the detector and at every $10^{\text {th }}$ frame, $\tilde{\operatorname{Pr}}(O N)$ and $\operatorname{Pr}(O F F)$ are estimated accordingly as given below:

$$
\begin{aligned}
\operatorname{Pr}(O N) & =\frac{\text { Number of decisions for } H_{1}}{10} \\
\operatorname{Pr}(O F F) & =\frac{\text { Number of decisions for } H_{0}}{10} .
\end{aligned}
$$

This way, the receiver makes the decision on the PU's states and consequently, takes the most probable value of the three, for $t_{s}$. The sensing time then remains constant for the rest of the next 10 frames, until the next estimation is performed.

\section{ALGORITHM DESCRIPTION AND EXPERIMENTAL SETUP}

\section{ALGORITHM}

The logic, which drives the cyclostationary sensing process is shown in Figure 1. After the measurement is performed, the algorithm calculates the variance of the signal power from the gathered samples and the decision threshold. Then, the CAF is calculated, the decision for the spectrum occupancy is made and if the spectrum is occupied, the algorithm will pause and wait until the end of the frame. In the alternative case, the same action will be taken because no actual transmission capabilities are implemented in the receiver (such are not necessary because only the local spectrum sensing scenario is studied in this article). The difference is, however, that in this case, the period of the interruption will be saved, as a transmission period of the $\mathrm{SU}$, together with the rest of the parameters. Finally, the estimation of the channel attenuation is done, followed by the calculation of the probability of detection. The algorithm of the energy detector spectrum sensing program follows the same pattern but goes through the specific steps of the method, as described in the previous section.

\section{MEASUREMENT TESTBED AND SOFTWARE}

The experiment was performed with the USRP hardware platform and the GNU Radio software, which gives the opportunity for intu- 




Figure 1.

Basic description of the operation of the algorithm within the span of one frame.

itive operation with the equipment. The USRP incorporates two elements a motherboard and a daughterboard. Our variant is USRP2. The motherboard consists of two digital-to-analog converters, which can produce up to $400 \mathrm{MS} / \mathrm{s}$, two analog-to-digital converters with sampling rate up to $100 \mathrm{MS} / \mathrm{s}$, programmable digital upand down-converters, a Gigabit Ethernet Interface, through which the USRP connects to the host computer. There is also a possibility for processing bandwidths of up to $100 \mathrm{MHz}$, and for operating with two antennas [43]. The daughterboard variant is XCVR2450, which is a half-duplex transceiver. It supports the 2.4- and 5-GHz bands and has a typical output power of $100 \mathrm{~mW}$ [44].

GNU Radio [45] provides the tools, which allow for many different kinds of transceivers to be easily constructed and then implemented with the USRP. In the recent versions, the usual installation under Linux includes everything that is needed for practical experiments. The drivers, which operate the equipment directly are written in $\mathrm{C}++$, but for easier operation, they are connected with Python scripts via the Simplified Wrapper and Interface Generator. In this way, the functions can be incorporated into blocks, which can be used for building the transceivers. The GNU Radio also provides a graphical user interface (GUI), which simplifies the process because it resembles Simulink. However, in cases such as

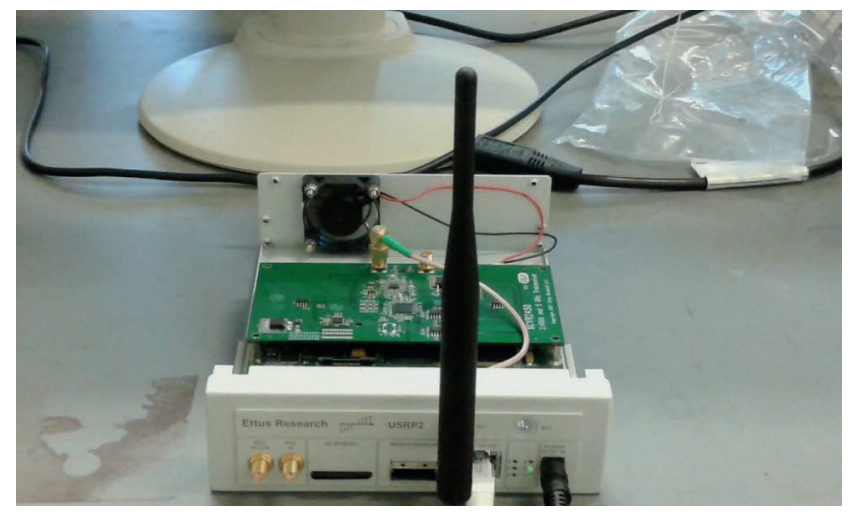

Figure 2.

USRP hardware platform. ours, it is easier and faster to write the whole logic of an algorithm in Python, without using the GUI editor.

\section{EXPERIMENTAL SETIP AND IMPLEMENTATION CHARACTERISTIES}

\section{MEASUREMENT SETUP}

Our experiment involves two USRPs (see, Figure 2), which are several meters apart on two tables close to each other. There are also obstacles, which form an NLOS scenario (see, Figure 3). One of them implements the PU transmitter $\left(T_{x}\right)$, while the other is the $\mathrm{SU}$ receiver $\left(R_{x}\right)$, which performs the spectrum sensing function.

For our experiment, we used two types of signals an OFDM and a direct spread spectrum (DSS). The DSS transmitter is built using the IEEE 802.15.4 OQPSK PHY block from the IEEE 802.15.4 Transceiver package for GNU Radio [46]. Whereas, the OFDM signal is created with the help of the OFDM Modulator block, which is a part of the gr-digital package [45]. Both of the transmitters are constructed using the GNU Radio editor, which produces the respective Python files. They are modified in order to implement the discontinuous transmission.

The PU transmits intermittently for randomly chosen periods of time. The same holds for the time while it is inactive. The periods during, which the PU is idle/active are taken preliminary from an exponential distribution so that its states may also be exponentially distributed, according to the model we assumed in the previous System Model section. By varying the upper bound on the two sets of time periods, we obtain the three different PU configurations as noted in the System Model section. Each of them runs for $300 \mathrm{sec}-$ onds and the resulting parameters are calculated by taking the sum of the active (and the idle, respectively) period and divide it by the whole runtime of the transmitter (to obtain $\operatorname{Pr}(O N)$ and $\operatorname{Pr}(O N)$ ), and by finding its mean (for $\mu_{O N}^{-1}$ and $\mu_{O F F}^{-1}$ ). The corresponding parameters for all three transmitters are presented in Table 1. On the basis of these parameters, the optimal sensing times for each configuration are obtained in the way described in the System Model section. In general, the transmitter program implements all three of the PU configurations, one after the other, so its complete execution time is 900 seconds. The operating frequency is $5 \mathrm{GHz}$, the bandwidth is $200 \mathrm{kHz}$, and the gain is $13 \mathrm{~dB}$.

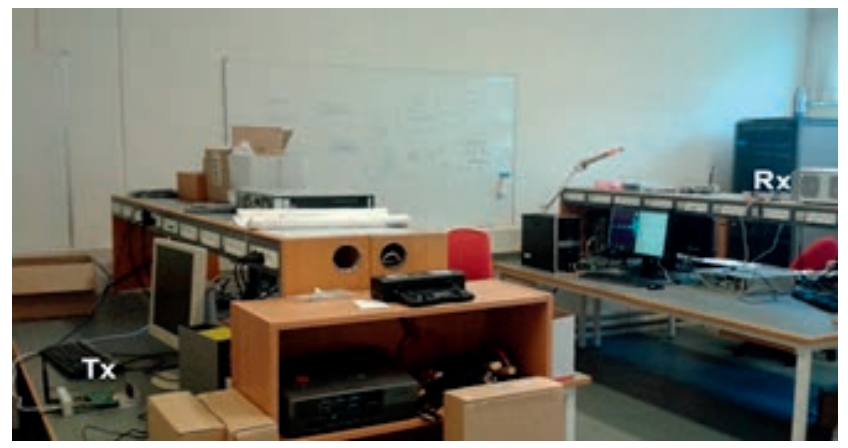

Figure 3.

Experimental Setup. 
Table 1.

\begin{tabular}{|c|c|c|c|c|c|}
\hline & $\operatorname{Pr}(O N)$ & $\operatorname{Pr}(O F F)$ & $\mu_{\text {ON }}^{-1}$ & $\mu_{\text {OFF }}^{-1}$ & $t_{s^{\prime}} \mathrm{ms}$ \\
\hline $\operatorname{Pr}(O N)>>\operatorname{Pr}(O F F)$ & 0.85 & 0.15 & 657 & 117 & 1.9 \\
\hline $\operatorname{Pr}(O N) \approx \operatorname{Pr}(O F F)$ & 0.42 & 0.58 & 267 & 328 & 6.6 \\
\hline $\operatorname{Pr}(O N) \ll \operatorname{Pr}(O F F)$ & 0.14 & 0.86 & 178 & 1,078 & 4.5 \\
\hline
\end{tabular}

We realize the SU receiver in a similar manner to the PU transmitter. The input samples are filtered with the FFT block and are then sent to the Message Sink, which allows for their extraction into a list that is processed inside a Python script. In this way, we obtain the complex data, which we use for the CAF calculation in the cyclostationary detector. For obtaining the signal variance and the channel estimation we use the squared magnitude of the samples. In the case of the energy detector, we only need the latter. The processing in Python is performed by the spectrum sensing algorithm as described previously. The radio-frequency parameters of the receiver are the same as those of the transmitter with the exception of the gain which is $16.6 / 19 / 18 \mathrm{~dB}$ (for the direct-sequence spread spectrum cyclostationary detector, OFDM cyclostationary detector, and the energy detector, respectively) and the addition of the FFT size equal to 64 .

In order to study the scenario of an environment where the $\mathrm{SU}$ devices have limited mobility within the area covered by the PU transmitter, the receiver is randomly moved by hand in the span of 2 meters from its initial position on the table.

\section{IMPLEMENTATION CONSIDERATIONS}

In the case of a practical application of spectrum sensing in an experimental environment, there are some considerations which may not exist when the study is done via computer simulation. We outline them briefly in the following.

An important thing to be regarded is the gain calibration of the USRP-based transmitter and receiver. The purpose is to define their gains so that the signal can be detected in the specific premise of the experiment. This is especially important in scenarios like ours where the transmitter and the receiver are not close to each other, and there is no line of sight. If the gains are not sufficiently large, the detector will not be able to differentiate the signal from the noise because the received samples will be too weak. On the other hand, in case the amplification is too high, we will not be able to study signals in low SNR conditions. In this implementation we set the gain by testing whether the PU signal is detected while the transmitter operates without interruptions. Thus, the best balance between the transmitter and the receiver gains is found empirically.

For the purpose of our study we examine a cyclostationary detector, which requires the cyclic frequency $\alpha$ and the time lag $\tau$, to be known. Even though, it is possible to assume their values using our general knowledge of the modulation of the PU signal, here we use the conclusions of some studies [47], [48] which pro- pose more realistic approaches to this problem. We first consider the analysis of the structure of different types of signals, made in [47]. This paper proposes that the DSS signal can be detected for $\alpha=f_{s}$ and $\tau=0$, because if we only want to examine whether the spectrum is occupied by such a kind of a transmission, we do not need to identify its components (the different users whose signals are spread along the bandwidth). As for the OFDM signal, $\tau=1 /$ $\Delta f\left(\Delta f\right.$ is the subcarrier separation) and $\alpha=k f_{s}(k \in \mathbb{R})$ because of its multicarrier structure. Generally, $k$ or the number of the CFs has not clearly been defined in most of the literature [8], [9], [36], [37], [27], in the sense that the question arises about how many and which values of $\alpha$ are to be assumed. Even though some of them can be acquired from the conclusions of [49], we utilize the empirical approach described in [48]. By plotting the cyclic correlogram of the signal we found that the strongest peak is around $0.2 f_{s}$ so this value of $\alpha$ was chosen. For greater computational efficiency of the cyclostationary method, we implement only single-cycle detection (CAF is calculated only for one $\alpha$ ). The correlogram is obtained by calculating the square magnitude of the CAF summed over all the observed samples for an initial value of $\alpha$ set to $f_{s}$.

In general, when measurements over the air are performed with the common USRP scenario (one being a transmitter, the other receiver) [4]-[9], the estimation of the received SNR has to be carefully considered. That is due to the need of assessing the noise variance (and also the nominal noise power) experimentally when the transmitter is inactive. For that reason, obtaining a very low SNR $(<<0 \mathrm{~dB})$ at the receiver, is not a trivial task. The authors in [7] propose for the received SNR to be defined as a fraction of the reference SNR level (called full scale SNR) which is the level measured when the transmitter and receiver are very close to each other. Using this method, the study in [7] is performed for SNR even less than $-15 \mathrm{~dB}$. That is why we utilize it in our experiment to define the SNR. The noise power is estimated by measuring the channel for 5 seconds in the absence of the transmitter's signal and finding the average of the 2,000,000 samples obtained.

Generally, the USRP receiver experiences local oscillator leakage which is dealt by using a Blackmann-Harris window function in the FFT block as it is also suggested in [50].

Considering the need for solving the Marcum $\mathrm{Q}$ function of first order [51], we suggest the algorithmic implementation proposed in [52]. It is not only easy to implement but also allows the use of the quad function (a part of the SciPy package which provides many useful functions for Python [53]) in order to solve the integral in (6). From purely computational point of view, an increase 
in the processing speed can be achieved by utilizing a more suitable programming language. In our implementation, we use Cython [54] to realize the slowest operations in the spectrum sensing program (like calculation of the $\mathrm{CAF}$ and solving integrals). By doing this, we do not need to change the Python implementation of these functions drastically, because Cython is based on Python. What it does is to translate the code into $\mathrm{C} / \mathrm{C}++$ and compile it. In this way the necessary complex mathematical operations can be executed within the Python runtime environment but use the speed advantage of a compiled $\mathrm{C}$ code [55]. The individual functions can be written in Python with minor modifications (defining some variables and functions as $\mathrm{C}$ variables and functions [56]) into a separate file and compiled. They are then imported into the main Python script and they are performed significantly faster.

Finally, when implementing a spectrum sensing framework, it is necessary to define the length of the frames. In this

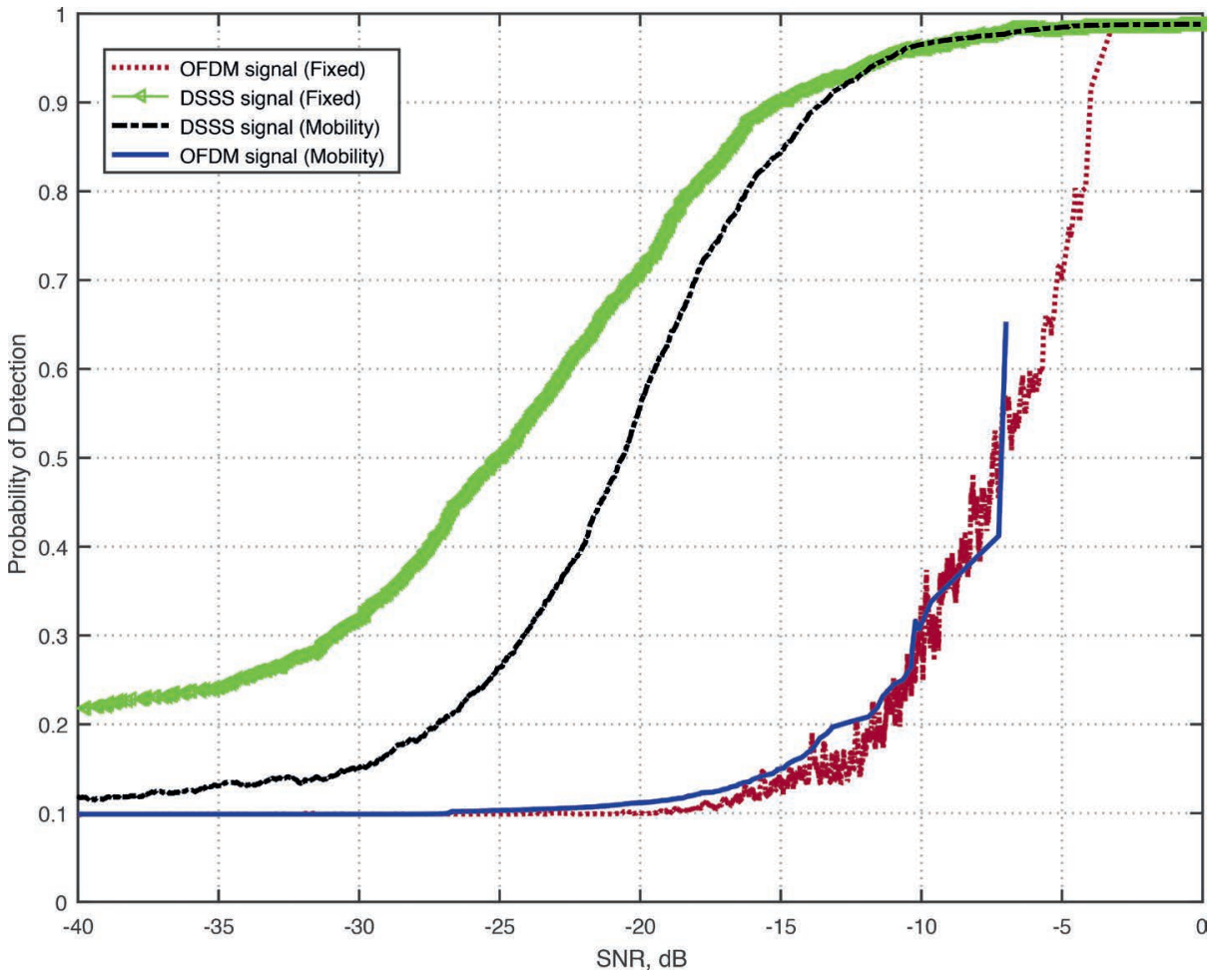

Figure 4.

Probability of Detection versus SNR for the Cyclostationary Detector in Fixed and Mobility cases. work we choose it as $10 \mathrm{~ms}$, taking into

consideration the following. First of all, the method of conducting the measurements with the USRP and GNU Radio allows for small sensing times. According to the IEEE 802.22 standard for cognitive radio networks [57], the size of the frame is also $10 \mathrm{~ms}$. There are also some studies, which examined spectrum sensing times in the range between 1 and $10 \mathrm{~ms}$ [23], [26], [58], [59].

\section{ANALYSIS OF THE RESULTS}

In this section we analyze the results for the parameters, by which we assess the performance of our practical implementation in terms of detection accuracy and efficiency of the estimator of the PU states. As stated in last section, we use two different PU transmitters for the cyclostationary detector and we compare its operation for both of them, together with that of the energy detector (which has no regard for the type of the PU signal). A comparison is also made for the fixed and mobility scenarios for the SU receiver. The target values for the probabilities of detection and of false alarm are 0.9 and 0.1 , respectively [57]. Figure 4 shows the distributions of the probabilities of detection of the cyclostationary detector for the two kinds of signals (DSS and OFDM) in two cases Fixed (the receiver is stationary) and Mobility (the receiver is in motion). The produced graphics required significant smoothing because of the intense fluctuations of the probability of detection, which are a consequence of the realistic environment of our experiment. Such variations were not experienced in the Fixed OFDM signal case for the low SNR levels. This is also seen through the line being almost perfectly straight in this interval. For both signal types the difference between the probabilities of detection for the Fixed and Mobility cases is up to around $10 \%$ and decreases rapidly as the SNR increases. This is to show that slow motion in the span of limited distance from the transmitter (as when a person carries the CR device in the premises of the room), does not significantly degrade the detection quality of the cyclostationary detector in the normal SNR range, which it is required to operate in, by the standard ( $-20 \mathrm{~dB}$ and higher) [57]. Another aspect, which is evident from the figure, is that the performance of the detector is significantly lower for the OFDM signal. As explained, the DSS signal is assumed to have a zero time-lag and thus, in order to compute the autocorrelation, the same sample is multiplied by itself (see (2)). Therefore, the CAF will naturally be greater than that of the OFDM signal which has a time lag of 128 samples ( $\Delta f$ for our scenario is $3.125 \mathrm{kHz}$ and the sampling rate is $400 \mathrm{kHz}$ ). It can also be noted that the cyclostationary detector does not achieve the required by the standard [57] detection rate of 0.9 for SNR of $-20 \mathrm{~dB}$. The same can also be concluded from the simulation-based examination which proposed the detector [27]. In contrast to the results of [27], however, it can be seen that the detector reaches the desired probability of detection for lower SNR levels (around $-15 \mathrm{~dB}$ ) for the DSS signal case.

The performance of the energy detector is shown in Figure 5. From the analysis in [28] can be observed that the performance of this spectrum sensing method is determined decisively by the number of received samples (which is an inherent characteristic of this type of detectors). By varying the sensing time and the sampling frequency, the same pattern became evident in our experiments. Specific detection probability can be obtained for a certain SNR level by increasing the number of samples. For that reason, we compare the accuracy of the detector in two scenarios. The first 


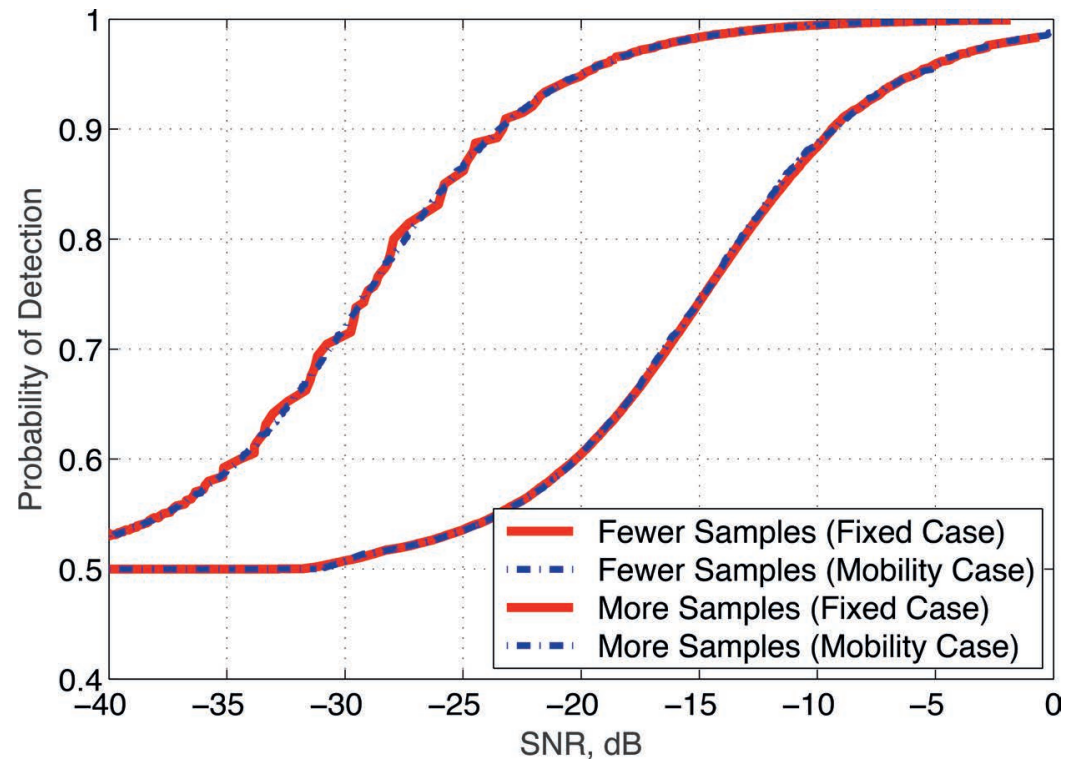

Figure 5.

Probability of Detection versus SNR for the Energy Detector in Fixed and Mobility cases in two scenarios for the number of samples.

one utilizes the same framework (and magnitude of the number of samples) as the cyclostationary detector, while the second one is realized by increasing the sampling rate and the sensing times of the receiver. Consequently, this requires changing the parameters of the PU as well. Under the "Fewer Samples" scenario, the detector analyzes at maximum of several thousand samples. That is why it is only able to reach the required probability of detection (0.9) at levels higher than $-10 \mathrm{~dB}$. As it was noted in [28], the detector needs more than 1.5 million samples to achieve the recommended detection rate in $-20 \mathrm{~dB}$ SNR under the standard-specific requirement for probability of false alarm (0.1). Despite of this, we reached this goal with just several hundred thousand samples in the "More Samples" scenario. The significant disadvantage of the second scenario is that the time to process so many samples increases by orders of magnitude. From the plots, it can be seen that for both of the scenarios, the curves start from detection rate of 0.5 or a little above it. The reason can be found by examining (11) with different values for the number of samples, the SNR and the ED decision threshold. Measurement trials have led us to believe that the probability of detection cannot fall beneath 0.5 in such SNR levels and the amount of observed samples.

To elaborate further, we perform similar analysis to the one in [28]. We set $\sigma_{\mathrm{w}}^{2}=1$, normalized threshold $\hat{\lambda}=\lambda / N$ and examine the range of possible values of the probability of detection for a fixed SNR in the interval of
$[0.95,1.15]$ for $\hat{\lambda}$ (Figure 6). The number of samples $N$ is 7,500 and 550,000 for the "Fewer Samples" and "More Samples" cases, respectively. It is of interest to us to analyze the values of $P_{d}$ for the respective SNR levels $(-40,-30$, and $-20 \mathrm{~dB})$ for $\hat{\lambda}=1$ (which corresponds to the actual threshold obtained by the bounded threshold selection). For SNR of $-40 \mathrm{~dB}$, this value is 0.5 for the "Fewer Samples" case and 0.53 for the "More Samples" case as shown in Figure 6 , respectively. This explains why the curves in Figure 5 start from these points.

As for the difference in the performance between the Fixed and the Mobility cases, we can observe that it is insignificant and therefore, we can make the same conclusion that we did for the cyclostationary detector.

We also assess the effectiveness of the PU states estimation scheme. Figure 7 shows the cumulative distribution functions of the estimated probability that the PU is in active state $\operatorname{Pr}(O N)$ of the PU transmitter and the two types of detectors. It is obvious that the detectors follow a similar to the original pattern of the distribution of the active states (marked in blue in Figure 7 but differing significantly. The detectors perceive the band as occupied for most of the time even though the pattern of transmission of the PU shows that in reality, it is otherwise (according to the settings of the PU configurations, defined in Experimental Setup and Implementation Characteristics). This is where the influence of the processing time becomes evident. In this experiment, it is not included into the analysis of the sensing time and its impact on the operation of the framework is not

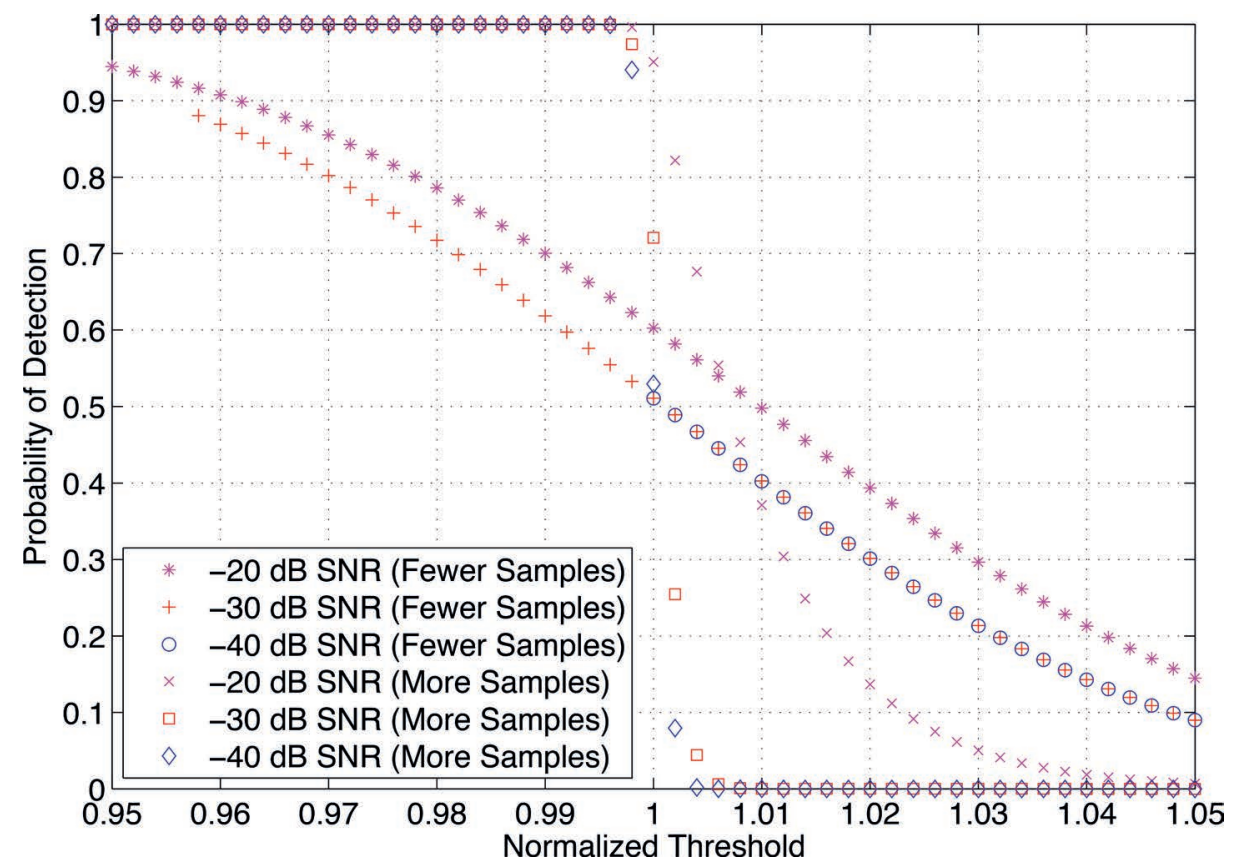

Figure 6.

Probability of Detection versus the normalized threshold for fixed SNR levels in two scenarios for the number of samples. 
considered in-depth. Even though, measures of reducing it have been taken (as explained in the Experimental Study section), the processing time causes the detector to miss sometimes the idle period of the PU and thus, it will detect the transmitter once more in the next sensing iteration. This will result in increasing of $\tilde{\operatorname{Pr}}(O N)$. The opposite can also occur. Another thing to be noted is that the transmitter program does not change from one state to the other instantly. The delay is due to the fact that the GNU Radio flow-graph which conducts the transmission process requires between a few and several tens of milliseconds to execute and to stop. Therefore, the transmitter also contributes to the inaccuracies in the PU states estimation.

\section{CONCLUSIONS AND FUTURE WORK}

In this article, we proposed an adaptive approach in the spectrum sensing process for improving the performance of existing cyclostationary and energy detectors based on a trade-off between efficiency (sensing time) and accuracy. We introduce adaptivity in the spectrum sensing process as a means to optimize it. We evaluate it in a practical implementation scenario by studying the performance of a cyclostationary detector and an energy detector, both integrated into a framework, which determines the optimal sensing time and was realized in practice through the USRP2 hardware platform. The results we obtain are very close to or better than those from the simulations described in the papers which proposed the implemented spectrum sensing methods ([28], [27]). It is also shown that the limited mobility of the SU does not cause a substantial degradation in the performance of the detectors. Therefore, the implementation of these methods in non-stationary devices is feasible.

As for the efficiency-accuracy trade-off, it has been noted that there is no necessity for it to be obtained during each detection instance, but it can be calculated preliminary for specific PU parameters. That is because the result of the trade-off is not influenced by the measurements of the SU receiver but by the pattern of transmission of the PU. Therefore, accurate definition of that pattern is critical. In a scenario like ours, the parameters of the transmitter are known but if a realistic arbitrary PU is employed, this will make the estimation problem much more complex. Thus, there are two questions which can be posed. Should the estimation of the transmission parameters be done on the basis of preliminary measurements of the PU activity? Or should we use an estimation algorithm which is integrated into the detectors framework (like the one utilized in this study)? If the former approach is adopted, then the means of measuring the active/idle periods of the transmitter have to be determined because the parameters of the PU (P(ON) and $\mathrm{P}(\mathrm{OFF})$, and $\mu_{O N}^{-1}$ and $\left.\mu_{O F F}^{-1}\right)$ are based on them. In the second case, the receiver will have also to try to predict them based not only on the detection statistics but on the choice of sensing time as well. Therefore, it will be required to perform the trade-off calculation together with the estimation of the probabilities that the PU is active/idle.

As an alternative, on the focus of the spectrum sensing research could be the optimization of the detection solution itself so that it is executed as fast as possible and the trade-off between its accuracy and sensing time can be found empirically and set as a standard. In this way the estimation of the PUs pattern of transmission will not be necessary.

Concerning the processing time of the solution, it also plays a role as we have concluded earlier. The cyclostationary detector processes the obtained samples several times slower than the energy detector in the equivalent scenario. However, it provides much greater detection accuracy in the DSS signal case but not when OFDM signal is applied. The energy detector improves its probability of detection with the number of samples but the time to process them also increases significantly (it is, on average, about 30 times greater than the computation time of the cyclostationary detector). Nevertheless, it fulfills the requirements of the standard [57] in terms of speed as well, because sensing periods for up to 2 seconds are permitted. It is then clear that both of the sensing methods perform well in low SNR but do not reach the specifications in [57] (detection probability of 0.9 at $-20 \mathrm{~dB}$
Figure 7.

Cumulative distributions functions of the estimated probability that the primary user is active in comparison to that of the transmitter. 
SNR) when the number of samples is as small as several thousand. Therefore, if larger sensing periods are considered, this disadvantage can be overcome but at the same time it should be remembered that the processing time will also increase with the quantity of the samples. These conclusions also give more meaning to the need for future analysis of practical implementations of other spectrum sensing techniques (like matched filter or wavelet detector), and of the tolerable balance between the overall processing time and the required detection performance.

\section{REFERENCES}

[1] Patil, K., Prasad, R., and Skouby, K. A survey of worldwide spectrum occupancy measurement campaigns for cognitive radio. In Proceedings of the IEEE 2011 International Conference on Devices and Communications (ICDeCom), 2011, 1-5.

[2] Guiducci, D., Carciofi, C., Petrini, V., Pompei, S., Llorente, J., Ferrer, V., et al. Sharing under licensed shared access in a live LTE network in the 2.3-2.4 GHz band end-to-end architecture and compliance results. In Proceedings of the 2017 IEEE International Symposium on Dynamic Spectrum Access Networks (DySPAN), 2017, 1-10.

[3] Chen, K.-C., and Prasad, R. Cognitive Radio Networks. Wiley: 2009.

[4] Rashid, R. A., Sarijari, M. A., Fisal, N., Lo, A., Yusof, S., Kamilah, S., et al. Spectrum sensing measurement using GNU radio and USRP software radio platform. In Proceedings of the Seventh International Conference on Wireless and Mobile Communications, 2011, 237-242.

[5] Younis, A., Cushman, I., Rawat, D., and Bista, B. B. Adaptive threshold based combined energy and spectrum-width detection for RF channel sensing in cognitive networks using USRP B200 GNU radios: An experimental study. In Proceedings of the 2016 IEEE SoutheastCon, 2016, 1-7.

[6] See, W. X., Syed-Yusof, S. K., and Rahim, M. K. A. Edge energy detection using GNU radio and USRP2: An analysis study. In Proceedings of the 2013 IEEE Malaysia International Conference on Communications (MICC), 2013, 87-91.

[7] Blad, A., Axell, E., and Larsson, E. G. Spectrum sensing of OFDM signals in the presence of CFO: New algorithms and empirical evaluation using USRP. In Proceedings of the 2012 IEEE 13th International Workshop on Signal Processing Advances in Wireless Communications (SPAWC), 2012, 159-163.

[8] Nafkha, A., Aziz, B., Naoues, M., and Kliks, A. Cyclostationaritybased versus eigenvalues-based algorithms for spectrum sensing in cognitive radio systems: Experimental evaluation using GNU radio and USRP. In Proceedings of the 2015 IEEE 11th International Conference on Wireless and Mobile Computing, Networking and Communications (WiMob), 2015, 310-315.

[9] Nafkha, A., Naoues, M., Cichon, K., and Kliks, A. Experimental spectrum sensing measurements using USRP Software Radio platform and GNU-radio. In Proceedings of the 2014 IEEE 9th International Conference on Cognitive Radio Oriented Wireless Networks and Communications (CROWNCOM), 2014, 429-434.

[10] Dobre, E.-I., Marţian, A., and Vlădeanu, C. USRP-based experimental platform for energy detection in cognitive radio systems. In Proceedings of the 2016 IEEE International Conference on Communications (COMM), 2016, 185-188.

[11] Tchobanova, A. N. Z., and Marinova, G. Implementation of a energy detection based cooperative spectrum sensing on USRP platform for a cognitive radio networks. In Proceedings of the IEEE Twelfth Advanced International Conference on Telecommunications (AICT2016), 2016.

[12] Kosunen, M., Turunen, V., Kokkinen, K., and Ryynanen, J. Survey and analysis of cyclostationary signal detector implementations on FPGA. IEEE Journal on Emerging and Selected Topics in Circuits and Systems, Vol. 3, 4 (2013), 541-551.

[13] Allan, D., Crockett, L., Weiss, S., Stuart, K., and Stewart, R. W. FPGA implementation of a cyclostationary detector for OFDM signals. In Proceedings of the 2016 IEEE 24th European Signal Processing Conference (EUSIPCO), 2016, 647-651.

[14] Ishwerya, P., Geethu, S., and Lakshminarayanan, G. Autocorrelation based spectrum sensing architecture on FPGA with dynamic offset compensation. In Proceedings of the IEEE Distributed Computing, VLSI, Electrical Circuits and Robotics (DISCOVER), 2016, 153-157.

[15] Spooner, C. M., Mody, A. N., Chuang, J., and Anthony, M. P. Tunnelized cyclostationary signal processing: A novel approach to lowenergy spectrum sensing. In Proceedings of the 2013 IEEE Military Communications Conference (MILCOM 2013)-, 2013, 811-816.

[16] Papadakis, S., Surligas, M., Triantafyllakis, K., Vardakis, G., Gkiolias, A., Karamolegos, N., et al. An agile OFDM cognitive radio engine. In Proceedings of the 2017 IEEE International Symposium on Dynamic Spectrum Access Networks (DySPAN), 2017, 1-2.

[17] Kim, C. W., Ryoo, J., and Buddhikot, M. M. Design and implementation of an end-to-end architecture for $3.5 \mathrm{GHz}$ shared spectrum. In Proceedings of the 2015 IEEE International Symposium on Dynamic Spectrum Access Networks (DySPAN), 2015, 23-34.

[18] Agarwal, S., and De, S. Cognitive multihoming system for energy and cost aware video transmission. IEEE Transactions on Cognitive Communications and Networking, Vol. 2, 3 (2016), 316-329.

[19] Safavi-Naeini, H.-A., Roy, S., and Ashrafi, S. Spectrum sharing of radar and Wi-Fi networks: The sensing/throughput tradeoff. IEEE Transactions on Cognitive Communications and Networking, Vol. 1, 4 (2015), 372-382.

[20] Vosoughi, A., Cavallaro, J. R., and Marshall, A. Trust-aware consensus-inspired distributed cooperative spectrum sensing for cognitive radio ad hoc networks. IEEE Transactions on Cognitive Communications and Networking, Vol. 2, 1 (2016), 24-37.

[21] Lagunas, E., Sharma, S. K., Maleki, S., Chatzinotas, S., and Ottersten, B. Resource allocation for cognitive satellite communications with incumbent terrestrial networks. IEEE Transactions on Cognitive Communications and Networking, Vol. 1, 3 (2015), 305-317.

[22] Wujian, S., Weiguo, D., Lei, Z., Yang, L., and Ou, L. Efficiency-accuracy trade-off for spectrum sensing in cognitive network. In Proceedings of the 2012 IEEE Second International Conference on Instrumentation, Measurement, Computer, Communication and Control (IMCCC), 2012, 1136-1141.

[23] Liang, Y.-C., Zeng, Y., Peh, E. C., and Hoang, A. T. Sensing-throughput tradeoff for cognitive radio networks. IEEE Transactions on Wireless Communications, Vol. 7, 4 (2008), 1326-1337.

[24] Lee, W.-Y., and Akyildiz, I. F. Optimal spectrum sensing framework for cognitive radio networks. IEEE Transactions on Wireless Communications, Vol. 7, 10 (2008).

[25] Ivanov, A. Practical Implementation of Hybrid Accuracy-Time Spectrum Sensing for Cognitive Radio Networks, Aalborg University Library, 2016.

[26] Srisomboon, K., Prayote, A., and Lee, W. Two-stage spectrum sensing for cognitive radio under noise uncertainty. In Proceedings of the 
2015 IEEE Eighth International Conference on Mobile Computing and Ubiquitous Networking (ICMU), 2015, 19-24.

[27] Tani, A., Fantacci, R., and Marabissi, D. A low-complexity cyclostationary spectrum sensing for interference avoidance in Femtocell LTE-A-based networks. IEEE Transactions on Vehicular Technology, Vol. 65, 4 (2016), 2747-2753.

[28] Atapattu, S., Tellambura, C., Jiang, H., and Rajatheva, N. Unified analysis of low-SNR energy detection and threshold selection. IEEE Transactions on Vehicular Technology, Vol. 64, 11 (2015), 5006-5019.

[29] Plata, D. M. M., and Reátiga, Á. G. A. Evaluation of energy detection for spectrum sensing based on the dynamic selection of detectionthreshold. Procedia Engineering, Vol. 35, (2012), 135-143.

[30] Dubey, R. K., and Verma, G. Improved spectrum sensing for cognitive radio based on adaptive threshold. In Proceedings of the IEEE 2015 IEEE Second International Conference on Advances in Computing and Communication Engineering (ICACCE), 2015, 253-256.

[31] Bagwari, A., and Tomar, G. S. Two-stage detectors with multiple energy detectors and adaptive double threshold in cognitive radio networks. International Journal of Distributed Sensor Networks, 2013.

[32] Shrivastava, S., Tiwari, R., and Das, S. Comparative performance evaluation of a new dynamic-double-threshold energy detection scheme with basic spectrum sensing techniques. In Proceedings of the 2014 IEEE International Conference on Green Computing Communication and Electrical Engineering (ICGCCEE), 2014, 1-6.

[33] Khalaf, Z., Nafkha, A., and Palicot, J. Enhanced hybrid spectrum sensing architecture for cognitive radio equipment. In Proceedings of the 2011 IEEE XXXth URSI General Assembly and Scientific Symposium, 2011, 1-4.

[34] Hammadi, A. A., Alhussein, O., Sofotasios, P. C., Muhaidat, S., AlQutayri, M., Al-Araji, S., et al. Unified analysis of cooperative spectrum sensing over composite and generalized fading channels. IEEE Transactions on Vehicular Technology, Vol. 65, 9 (Sept. 2016), 6949-6961.

[35] Salvo Rossi, P., Ciuonzo, D., and Romano, G. Orthogonality and cooperation in collaborative spectrum sensing through MIMO decision fusion. IEEE Transactions on Wireless Communications, Vol. 12, 11 (2013), 5826-5836.

[36] Derakhshani, M., Le-Ngoc, T., and Nasiri-Kenari, M. Efficient cooperative cyclostationary spectrum sensing in cognitive radios at low SNR regimes. IEEE Transactions on Wireless Communications, Vol. 10, 11 (2011), 3754-3764.

[37] Bagwari, A., and Singh, B. Comparative performance evaluation of spectrum sensing techniques for cognitive radio networks. In Proceedings of the 2012 IEEE Fourth International Conference on Computational Intelligence and Communication Networks (CICN), 2012, 98-105.

[38] Han, D., and Liu, H. An energy detection based on cyclostationary. In Proceedings of the 2011 IEEE 7th International Conference on Wireless Communications, Networking and Mobile Computing (WiCOM), 2011, 1-4.

[39] Gifford, W. M., Win, M. Z., and Chiani, M. Diversity with practical channel estimation. IEEE Transactions on Wireless Communications, Vol. 4, 4 (2005), 1935-1947.

[40] Kaushik, A., Sharma, S. K., Chatzinotas, S., Ottersten, B., and Jondral, F. K. Sensing-throughput tradeoff for interweave cognitive radio system: A deployment-centric viewpoint. IEEE Transactions on Wireless Communications, Vol. 15, 5 (2016), 3690-3702.
[41] Urkowitz, H. Energy detection of unknown deterministic signals. Proceedings of the IEEE, Vol. 55, 4 (1967), 523-531.

[42] Haviv, M. Queues: A course in queueing theory. International Series in Operations Research \& Management Science 2013, Springer-Verlag New York, 21-36.

[43] Ettus Research. The USRP2 knowledge base page. Apr. 2017. [Online] Available: https://kb.ettus.com/USRP2.

[44] Ettus Research. XCVR2450 knowledge base page. Apr. 2017. [Online] Available: https://kb.ettus.com/USRP2.

[45] The GNU Radio Foundation. GNU Radio, the free and open software radio ecosystem. Apr. 2017. [Online] Available: http:// gnuradio.org.

[46] Bloessl, B., Leitner, C., Dressler, F., and Sommer, C. A GNU radiobased IEEE 802.15. 4 testbed. In Proceedings of the 12. GI/ITG KuVS Fachgespräch Drahtlose Sensornetze (FGSN 2013), 2013, $37-40$.

[47] Öner, M., and Jondral, F. Air interface identification for software radio systems. AEU-International Journal of Electronics and Communications, Vol. 61, 2 (2007), 104-117.

[48] Napolitano, A. Cyclostationarity: New trends and applications. Signal Processing, Vol. 120, (2016), 385-408.

[49] Gardner, W. A., and Spooner, C. M. Signal interception: Performance advantages of cyclic-feature detectors. IEEE Transactions on Communications, Vol. 40, 1 (1992), 149-159.

[50] Kim, J. Y., Marcum, A. C., Balmos, A. D., Layton, A. W., Larew, S. G., Krogmeier, J. V., et al. Implementation and analysis of energy detection-based sensing using USRP/SBX platform. In Proceedings of the 2014 IEEE Military Communications Conference (MILCOM), 2014, 1504-1509.

[51] András, S., Baricz, A., and Sun, Y. The generalized Marcum Q-function: An orthogonal polynomial approach. Acta Universitatis Sapientiae Mathematica, Vol. 3, 1 (2011), 60-76.

[52] McGee, W. Another recursive method of computing the Q function (Corresp.). IEEE Transactions on Information Theory, Vol. 16, 4 (1970), 500-501.

[53] SciPy. Scientific computing tools for Python. Apr. 2017. [Online] Available: http://scipy.org/.

[54] Bradshaw, R., Behnel, S., Seljebotn, D. S., Ewing, G. The Cython compiler. Apr. 2017. [Online] Available: http://cython.org.

[55] Behnel, S., Bradshaw, R. W., and Seljebotn, D. S. Cython tutorial. In Proceedings of the 8th Python in Science Conference, 2009, 4-14.

[56] Behnel, S., Bradshaw, R., Seljebotn, D. S., Ewing, G., Stein, W., Gellner, G., et al. Cython documentation. 2017. [Online] Available: https:// cython.readthedocs.io/en/latest/.

[57] Stevenson, C. R., Chouinard, G., Lei, Z., Hu, W., Shellhammer, S. J., and Caldwell, W. IEEE 802.22: The first cognitive radio wireless regional area network standard. IEEE Communications Magazine, Vol. 47, 1 (2009), 130-138.

[58] Bae, S., and Kim, H. Unlimited cooperative sensing with energy detection for cognitive radio. Journal of Communications and Networks, Vol. 16, 2 (2014), 172-182.

[59] Chye, Y. H., Dutkiewicz, E., Vesilo, R., and Liu, R. P. Adaptive spectrum sensing for cognitive radio systems in a fading environment. In Proceedings of the 2014 IEEE International Symposium on Wireless Personal Multimedia Communications (WPMC), 2014, 451-456. 